\title{
Magnetic field effect on tunnel ionization of deep impurities by terahertz radiation
}

\author{
S.D. Ganichev ${ }^{\mathrm{a}, \mathrm{b}, *}$, S.N. Danilov ${ }^{\mathrm{a}}$, M. Sollinger ${ }^{\mathrm{b}}$, J. Zimmermann ${ }^{\mathrm{a}}$, \\ A.S. Moskalenko ${ }^{\text {, }, ~ V . I . ~ P e r e l ~}{ }^{\mathrm{b}}$, I.N. Yassievich ${ }^{\mathrm{b}}$, C. Back ${ }^{\mathrm{a}}$, W. Prettl ${ }^{\mathrm{a}}$ \\ ${ }^{a}$ Institut für Exp. und Angew. Physik, Universität Regensburg, D 93040 Regensburg, Germany \\ ${ }^{\mathrm{b}}$ A.F.Ioffe Physico-Technical Institute, St. Petersburg, Russia
}

\begin{abstract}
A suppression of tunnelling ionization of deep impurities in terahertz frequency electric fields by a magnetic field is observed. It is shown that the ionization probability at external magnetic field, $\boldsymbol{B}$, oriented perpendicular to the electric field of terahertz radiation, $\boldsymbol{E}$, is substantially smaller than that at $\boldsymbol{B} \| \boldsymbol{E}$. The effect occurs at low temperatures and high magnetic fields.
\end{abstract}

(C) 2003 Elsevier B.V. All rights reserved.

PACS: 71.55.Eq; 72.10.Fk; 73.40.Gk

Keywords: Tunnel ionization; Terahertz radiation; Deep centers; Magnetic field

\section{Overview}

Tunneling ionization of deep centers in a terahertz field of high-intensity far-infrared laser radiation, with photon energies tens of times lower than the impurity binding energies has been investigated in great detail during the last decade [1]. In contrast to tunneling ionization of atoms, where only electron tunneling takes place, ionization of impurities in solids is accomplished by two simultaneous processes: electron tunneling through the potential well formed by the attractive force of the impurity and the externally applied electric field and the redistribution of the vibra-

\footnotetext{
*Corresponding author. Tel.: + 49-941-943-2050; fax: +49941-943-4223.

E-mail address: sergey.ganichev@physik.uni-regensburg.de (S.D. Ganichev).
}

tional system by defect tunneling. At very high radiation electric field strengths direct tunneling may occur without involving phonons.

The tunneling probability is independent on the radiation frequency up to very high frequencies. In this quasi-static regime, electrons tunnel at constant energy in a time much shorter than the reciprocal radiation frequency $\omega^{-1}$. At higher frequencies, however, tunneling probability drastically increases in comparison to the tunneling in DC field of the same field strength. In such a highfrequency regime electrons can absorb energy from the radiation field during tunneling leaving the barrier at higher energy. By this the effective width of the tunneling barrier is reduced and, thus, the tunneling probability enhanced.

In a semiclassical approach defect tunneling takes place from the adiabatic potential corresponding to the impurity bound state to the state 
where the carrier is detached from the impurity. The tunneling probability is controlled by the Büttiker-Landauer tunneling time [2] $\tau$ which is a function of temperature. The transition from frequency-independent tunneling in a classical electric field to fully quantized multi-photon transitions occurs in the terahertz range and may be explored applying high-power far-infrared lasers. The borderline is given by $\omega \tau=1$ where $\omega$ is the radiation frequency.

These considerations are based on semiclassical theory where the carriers have a classical trajectory. In this case the tunneling probability is expected to be affected by the strength and the orientation of an external magnetic field. For electron tunneling through static potential barriers this effect was theoretically investigated in Ref. [3] and observed in quantum well structures [4]. The theory has been extended for phonon-assisted tunneling ionization of deep impurities in DC electric fields [5] and in high-frequency alternating fields $[6,7]$ showing that also in the case of phononassisted tunneling, even in the high-frequency regime, the carrier emission is suppressed by an external magnetic field $(\boldsymbol{B} \perp \boldsymbol{E})$. In this work, we give evidence for the applicability of the semiclassical model to tunneling assisted by phonons which is concluded from the experimental observation of the suppression of tunneling probability by an external magnetic field oriented perpendicular to the carrier trajectory.

\section{Tunneling in alternating fields}

We have shown in Ref. [1] that the probability of phonon assisted tunneling depends on the strength of $\boldsymbol{E}$, an oscillating electric field of frequency $\omega$ :

$e(E) \propto \exp \left[\frac{E^{2}}{\left(E_{\mathrm{c}}^{*}\right)^{2}}\right] \quad$ with $\quad\left(E_{\mathrm{c}}^{*}\right)^{2}=\frac{3 m^{*} \hbar}{e^{2}\left(\tau^{*}\right)^{3}}$,

with electron effective mass $m^{*}$ and

$\left(\tau^{*}\right)^{3}=\frac{3}{4 \omega^{3}}(\sinh (2 \omega \tau)-2 \omega \tau)$.

The frequency dependence of the tunneling process is controlled by $\omega \tau$ where the tunneling time $\tau$ depends on temperature: $\tau=\hbar / 2 k T \pm \tau_{1}$. Here $\tau_{1}$ is of the order of the period of the impurity vibration and plus and minus correspond to substitutional and auto-localized impurities, respectively [8]. It follows from Eq. (2) that for $\omega \tau \ll 1$ the effective time $\tau^{*}$ gets equal to the tunneling time $\tau$ yielding a well known formula of phonon assisted tunneling in the quasi-static regime [1].

In the presence of an external magnetic field oriented perpendicular to the electric field of radiation, the functional dependence of the probability on the electric field strength remains unchanged, however the value of the effective time $\tau^{*}$ becomes dependent on the magnetic field strength. An increase of the cyclotron frequency $\omega_{\mathrm{c}}=e B / m^{*}$ over the reciprocal tunneling time results in the decrease of the tunneling probability. The suppression of the tunneling probability occurs in both frequency ranges, at low frequencies, when tunneling is independent of radiation frequency, at high frequencies when the tunneling probability increases drastically with rising frequency. The effect of a magnetic field $\boldsymbol{B}$ on tunneling is strongest if it is oriented normal to the radiation electric field $\boldsymbol{E}$ and vanishes if $\boldsymbol{B} \| \boldsymbol{E}$. For the ionization probability we again obtain an exponential dependence on the square of the electric field strength in the form of Eq. (1), however, now the effective time $\tau^{*}$ depends on the magnetic field strength:

$$
\begin{aligned}
\left(\tau^{*}\right)^{3}= & \frac{3 \omega_{\mathrm{c}}^{2}}{\left(\omega^{2}-\omega_{\mathrm{c}}^{2}\right)^{2}} \\
& \times\left\{\int _ { 0 } ^ { \tau } \left(-\cosh \omega \tau^{\prime}\right.\right. \\
& \left.+\frac{\omega_{\mathrm{c}}}{\omega} \frac{\sinh \omega \tau}{\sinh \omega_{\mathrm{c}} \tau} \cosh \omega_{\mathrm{c}} \tau^{\prime}\right)^{2} \mathrm{~d} \tau^{\prime} \\
& +\int_{0}^{\tau}\left(\frac{\omega}{\omega_{\mathrm{c}}} \sinh \omega \tau^{\prime}\right. \\
& \left.\left.-\frac{\omega_{\mathrm{c}}}{\omega} \frac{\sinh \omega \tau}{\sinh \omega_{\mathrm{c}} \tau} \sinh \omega_{\mathrm{c}} \tau^{\prime}\right)^{2} \mathrm{~d} \tau^{\prime}\right\}
\end{aligned}
$$

In Fig. 1, the calculated impurity ionization probability is plotted as a function of $E^{2}$ for various magnetic field strengths showing the drop 
of the carrier emission rate with increasing $\boldsymbol{B}$. The data are presented for two different values of $\omega \tau$ representing the quasi-static and the highfrequency regimes.

\section{Experimental technique and results}

The experiments were carried out on mercury doped germanium in the temperature range of 10 $70 \mathrm{~K}$. Tunneling ionization has been achieved by far-infrared laser radiation with photon energies much smaller than the thermal impurity ionization energy $\varepsilon_{\mathrm{T}}=90 \mathrm{meV}$. The radiation source was a pulsed far-infrared molecular laser optically pumped by a TEA- $\mathrm{CO}_{2}$ laser. Operating the optically pumped laser with $\mathrm{NH}_{3}$ and $\mathrm{CH}_{3} \mathrm{~F}$ as active gases, $40 \mathrm{~ns}$ pulses with intensity up to $2 \mathrm{MW} / \mathrm{cm}^{2}$ have been obtained at wavelengths of 148 and $496 \mu \mathrm{m}$. The ionization probability has been measured by detecting photoconductivity [1]. To apply a small probe voltage to the sample two ohmic contacts along $x$ direction were prepared. An external magnetic field $\boldsymbol{B}$ up to $7.5 \mathrm{~T}$ has been applied along $x$. The ionization probability as a function of the radiation intensity $I \propto E^{2}$ has been obtained for $\boldsymbol{E} \| B_{x}$ and $\boldsymbol{E} \perp B_{x}$ for different magnetic field strengths. In addition, the magnetic

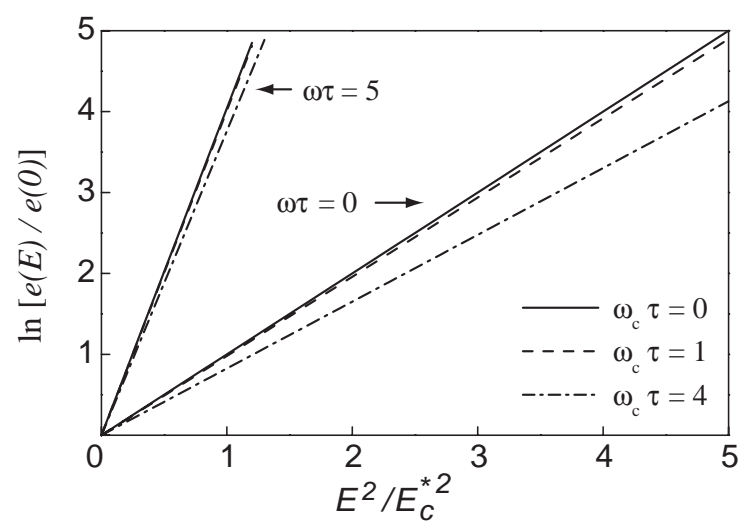

Fig. 1. $\ln [e(E) / e(0)]$ as a function of $E^{2} / E_{\mathrm{c}}^{* 2}$ for different $\omega \tau$ and $\omega_{\mathrm{c}} \tau$ calculated after Eqs. (1)-(3) for a magnetic field normal to the electric field; $\omega$ and $\omega_{\mathrm{c}}$ are the radiation frequency and the cyclotron frequency, respectively. Here the parameter $\omega \tau$ controls the frequency dependence of tunneling while $\omega_{\mathrm{c}} \tau$ reflects the influence of the magnetic field. field dependence of the signal has been determined for constant intensity. The orientation between the external magnetic field $B_{x}$ and the electric field $\boldsymbol{E}$ of linearly polarized terahertz radiation has been changed by means of a $\lambda / 2$ crystal quartz plate.

In Fig. 2, the dependence of the photoconductive signal being proportional to the ionization probability on the square of the electric field strength of the radiation is plotted for $B=0$ and 7.4 T. Results are presented for a temperature of $15 \mathrm{~K}$ and $\lambda=148 \mu \mathrm{m}$. These measurements show that at zero magnetic field the ionization probability is independent of the electric field orientation. At high magnetic fields, however, the signal for $\boldsymbol{E} \perp B_{x}$ drops significantly below that of $\boldsymbol{E} \| B_{x}$.

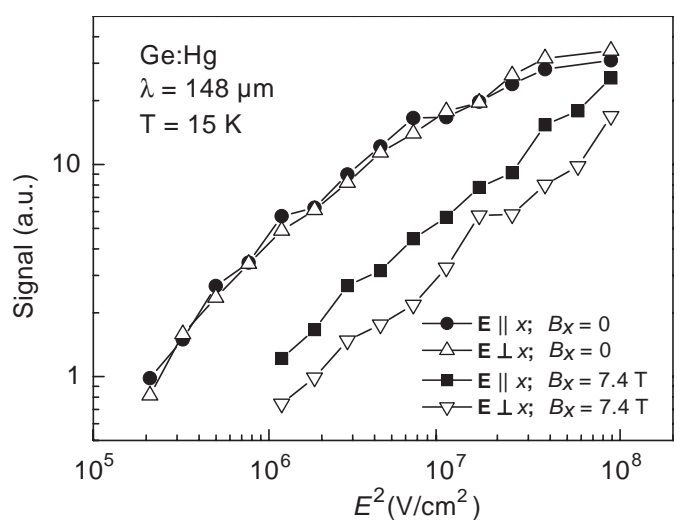

Fig. 2. Photoconductive signal for $\mathrm{Ge}: \mathrm{Hg}$ as a function of $E^{2}$ for different magnetic field strengths $B_{x}$ and orientations.

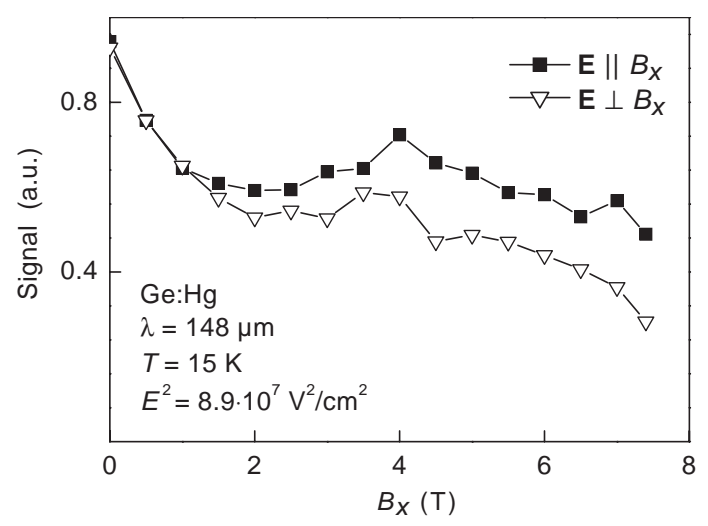

Fig. 3. Photoconductive signal as a function of magnetic field strength for two polarizations: $\boldsymbol{E} \perp B_{x}$ and $\boldsymbol{E} \| B_{x}$ in the highfrequency limit $(\omega \tau>1)$. 


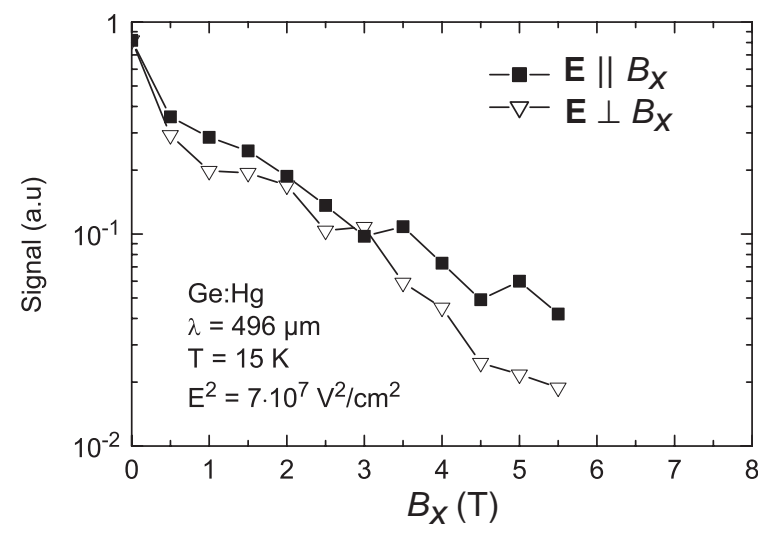

Fig. 4. Photoconductive signal as a function of magnetic field strength for two polarizations: $\boldsymbol{E} \perp B_{x}$ and $\boldsymbol{E} \| B_{x}$ in the quasistatic limit $(\omega \tau<1)$.

This suppression of the tunneling probability can also be seen in the magnetic field dependence of the photoconductive signal shown in Figs. 3 and 4. The effect of tunneling suppression occurs only at low temperatures where, on one side, practically all carriers are frozen out on the impurities and on the other side the tunneling time assumes high values [1]. At high temperatures the effect of free carrier absorption interferes with the change of conductivity by tunneling ionization. Therefore no suppression of the signal could be observed. Tunneling suppression has been observed in both regimes, the quasi-static limit $(\omega \tau<1)$ at $496 \mu \mathrm{m}$ (Fig. 3) and in the high-frequency limit $(\omega \tau>1)$ obtained by the excitation with radiation of $148 \mu \mathrm{m}$ (Fig. 4).

In summary, our present observation shows that for $\boldsymbol{B} \perp \boldsymbol{E}$ the magnetic field deflects the carriers, which increases the length of the tunneling trajectory. Thus, a magnetic field reduces the ionization probability if the cyclotron frequency becomes larger than the reciprocal tunneling time. Experimental findings are in good agreement with the developed theory of phonon-assisted tunneling ionization of deep impurities in the presence of a magnetic field.

\section{References}

[1] S.D. Ganichev, I.N. Yassievich, W. Prettl, J. Phys.: Condens. Matter 14 (2002) R1263.

[2] R. Landauer, Th. Martin, Rev. Mod. Phys. 66 (1994) 217.

[3] L.P. Kotova, A.M. Perelomov, V.S. Popov, Sov. JETP 27 (1968) 616.

[4] L. Eaves, K.W.H. Stevens, F.W. Sheard, in: M.J. Kelly, C. Weisbuch (Eds.), The Physics and Fabrication of Microstructures and Microdevices, Springer, Berlin, 1986, pp. 343-349.

[5] V.I. Perel, I.N. Yassievich, JETP Lett. 68 (1998) 804.

[6] A.S. Moskalenko, S.D. Ganichev, V.I. Perel, I.N. Yassievich, Physica B 273-274 (1999) 1007.

[7] A.S. Moskalenko, V.I. Perel, I.N. Yassievich, JETP 90 (2000) 217.

[8] S.D. Ganichev, J. Diener, I.N. Yassievich, W. Prettl, B.K. Meyer, K.W. Benz, Phys. Rev. Lett. 75 (1995) 1590. 